\title{
Acute Pancreatitis with Large Infected Pseudo Pancreatic Cyst in a Post-Partum Female - Case Report
}

\author{
Sameera Dronamrajuํㅜ, Yash Gupte², Twinkle Pawar ${ }^{3}$, Sourya Acharya ${ }^{4}$, Sunil Kumar ${ }^{5}$ \\ 1, 2, 3, 4,5 Department of Medicine, Datta Meghe Institute of Medical Sciences (Deemed to be University), \\ Jawahar Lal Nehru Medical College, Sawangi Meghe, Wardha, Maharashtra, India.
}

\section{INTRODUCTION}

A frequent cause for patient presentation to the emergency department and the most serious gastrointestinal condition resulting in admission is acute pancreatitis. Pancreatitis is an inflammatory process within the pancreas. Although the disease is mostly mild, the mortality rate of severe forms may be up to 30 percent. Two of the following three criteria are required for diagnosis: epigastric abdominal pain, elevated lipase, and pancreatic inflammation on imaging. ${ }^{1}$

The occurrence of acute pancreatitis is approximately 1 in 1000 to 5000 births and is commonly seen in the last weeks of gestation or in post-partum period. Cholelithiasis, which accounts for more than 65 percent of cases, is the most common cause of acute pancreatitis in pregnancy. ${ }^{2}$

Pancreatic ascites results from persistent leakage of pancreatic secretions in the peritoneum from pancreatic duct injury. The extent of pancreatic ascites varies, depending on the site and degree of ductal damage and infection. ${ }^{3}$

The complications of acute or chronic pancreatitis are pancreatic pseudocysts. Initial diagnosis is mostly done by imaging. Endoscopic ultrasound with fine needle aspiration cytology (FNAC) has become the standard test to help differentiate pseudocyst from other cystic lesions of the pancreas. With supportive treatment, most pseudocysts resolve spontaneously. Poor predictors for the potential of pseudocyst resolution or complications are the size of the pseudocyst and the length of time the cyst has been present, but larger cysts in general are more likely to be symptomatic or cause complications. ${ }^{4}$

We report a case of young female presenting with jaundice and ascites two days post-partum, who was eventually diagnosed as a case of pancreatic ascites with large pancreatic pseudocyst.

\section{PRESENTATION OF CASE}

A 33 - year - old female, day 7 full term vaginal delivery of parity two, presented with yellowish discolouration of sclera, vomiting, distension of abdomen, facial puffiness and bilateral oedema of feet for five days. Patient delivered vaginally at a private hospital on day 2 of her delivery, she started experiencing the above symptoms and was referred to our hospital for further management.

On examination, icterus, pallor, distention of abdomen with bilateral oedema of feet (pitting) were noted. On percussion of abdomen, dull note was heard all over abdomen, shifting dullness could be elicited. Patient was afebrile with pulse 132/min, SpO2 - $96 \%$ (on room air), and blood pressure was 110/70 mm of Hg.
Corresponding Author: Dr. Sameera Dronamraju, Flat No. 13, Meghe Heights 3, DMIMS, Wardha - 442001, Maharashtra, India. E-mail: sameeradronamraju1993gmail.com

DOI: $10.14260 / j e m d s / 2021 / 627$

How to Cite This Article: Dronamraju S, Gupte Y, Pawar T, et al. Acute pancreatitis with large infected pseudo pancreatic cyst in a post-partum female - case report. J Evolution Med Dent Sci 2021;10(35):3074-3076, DOI: 10.14260/jemds/2021/627

Submission 06-02-2021, Peer Review 26-07-2021, Acceptance 02-08-2021, Published 30-08-2021.

Copyright (C) 2021 Sameera Dronamraju et al. This is an open access article distributed under Creative Commons Attribution License [Attribution 4.0 International (CC BY 4.0)] 
Investigations - Complete blood count (CBC) - $\mathrm{Hb}-6.5 \mathrm{gm}$ $\%$, mean corpuscular volume (MCV) - $68.5 \mathrm{f} / \mathrm{l}$, total leukocyte count (TLC) $-53,600 / \mathrm{mm}^{3}$, of which neutrophils were $85 \%$, absolute platelet count $-35,000 / \mathrm{mm}^{3}$, lactate dehydrogenase (LDH) - $1320 \mathrm{U} / \mathrm{L}$, aspartate aminotransferase (AST) - 108 $\mathrm{U} / \mathrm{L}$, Total bilirubin - $9.8 \mathrm{mg} / \mathrm{dl}$, Total protein $-4.3 \mathrm{mg} / \mathrm{dl}$, Serum lipase - 780 U/L, Serum amylase - 1120 U/L.
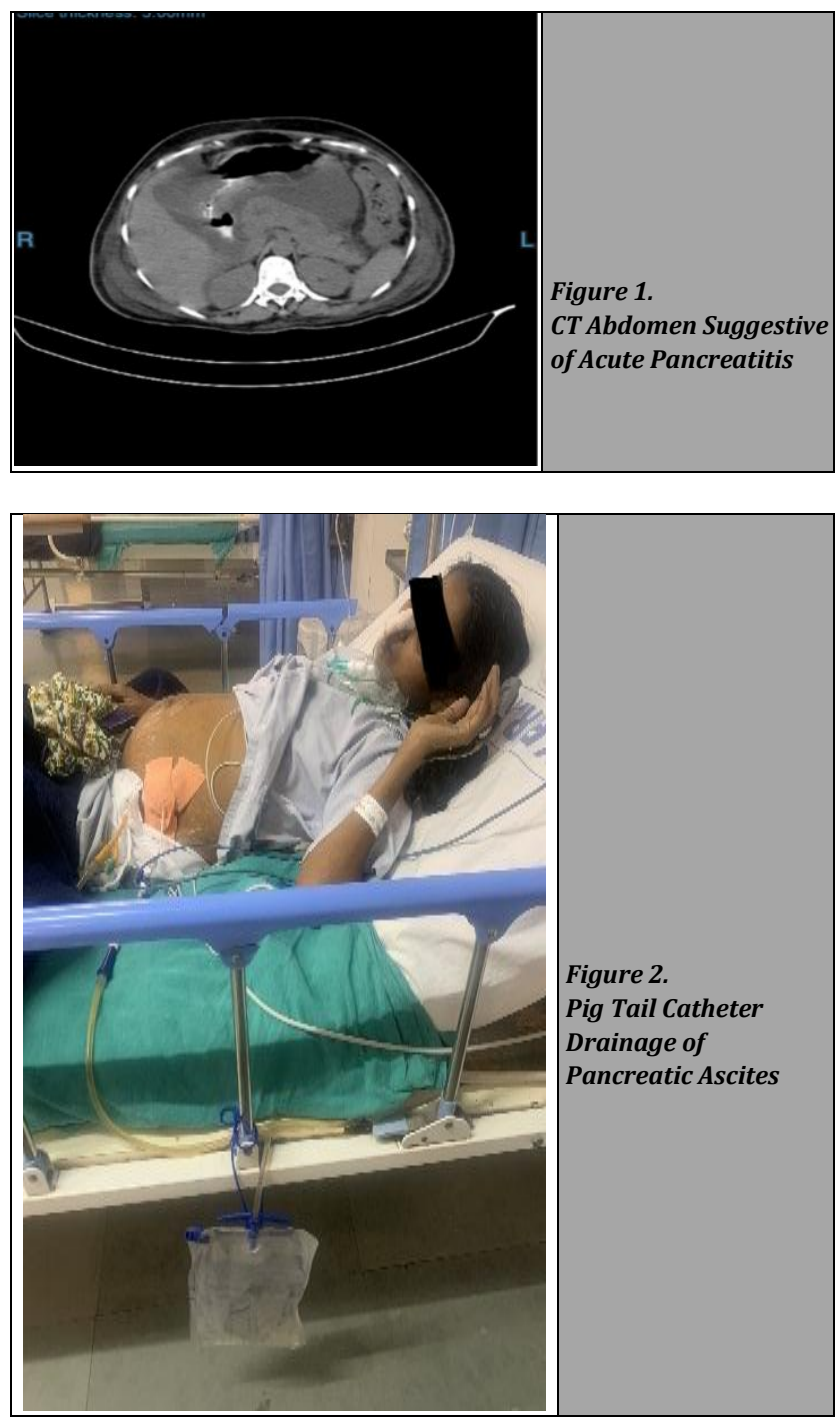

Fig 3 - CECT image suggestive of pseudo pancreatic cyst (white arrow). Fig 4. MRCP image of pancreatic pseudocyst causing mass effect on the pancreas, stomach. (blunt arrow)

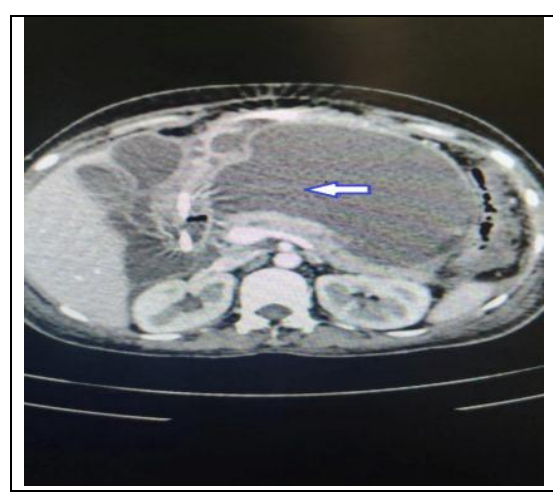

Figure 3.

CECT Image

Suggestive of Pseudo Pancreatic Cyst (Whit Arrow)

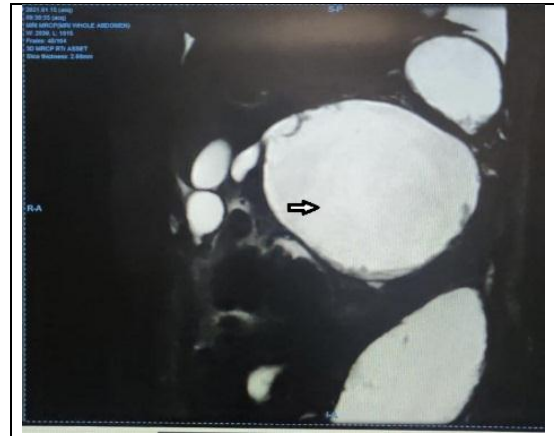

Figure 4.

MRCP Image of Pancreatic Pseudocyst Causing Mass Effect on the Pancreas, Stomach (Blunt arrow)

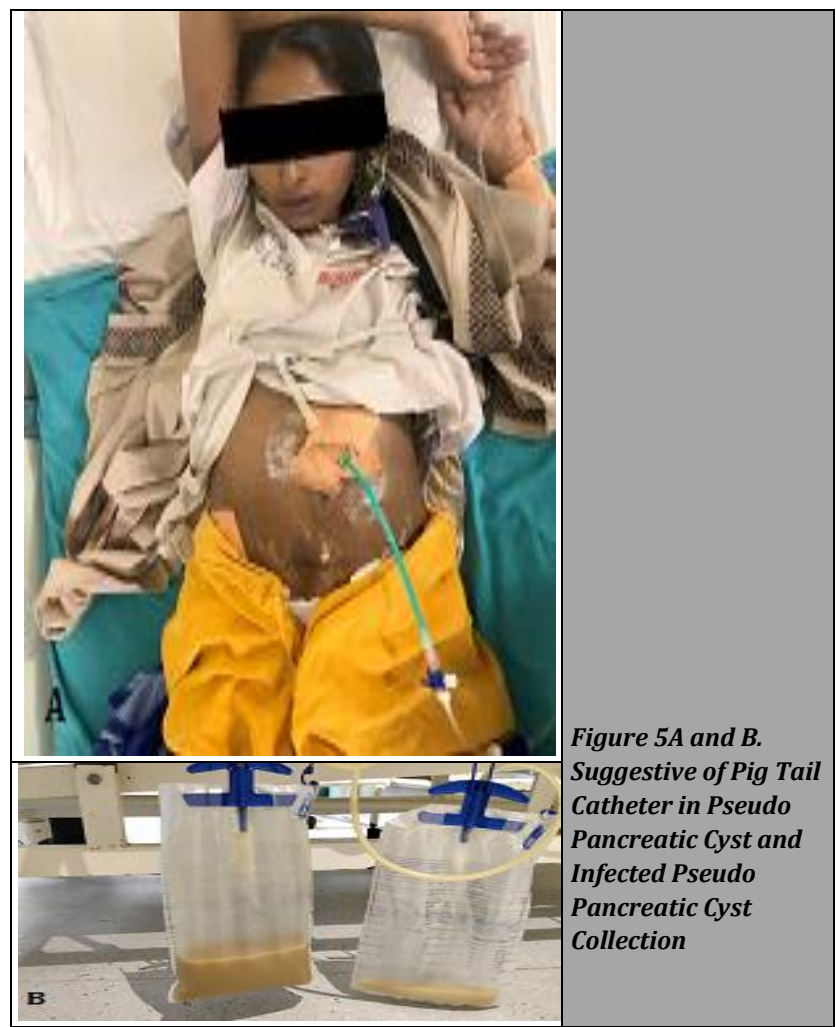

Ultrasonography (USG) of abdomen and pelvis was done which was suggestive of acute pancreatitis (bulky pancreas with multifocal patchy necrosis), and moderate ascites. Contrast enhanced computed tomography (CECT) of abdomen was done to confirm USG findings, findings were consistent with that of USG. (Fig. 1) Paracentesis was performed, both diagnostic and therapeutic. Ascitic fluid was sent for analysis in laboratory. Ascitic fluid analysis findings were confirmatory of pancreatitis and pancreatic ascites with ascitic fluid amylase - $8542 \mathrm{U} / \mathrm{L}$ and lipase was $3200 \mathrm{U} / \mathrm{L}$. Patient was started on treatment with higher antibiotics, intravenous fluids and other supportive care. She was kept nil by mouth until acute symptoms subsided. Pig tail catheter insertion was done for drainage of ascitic fluid. The patient continued to have refractory ascites even after 2 weeks of pigtail drainage. Repeat CECT of abdomen was done which was suggestive of well-defined cystic lesion in lesser sac displacing the stomach anteriorly, located anterior to the pancreas with well-defined thin wall, multiple collections noted in both paracolic gutters suggestive of infected collection. (Fig 3. A)

Magnetic resonance cholangiopancreatography (MRCP) was done, it was suggestive of acute pancreatitis with pseudo cyst communicating with main pancreatic duct, causing mass effect on the pancreas, stomach and left kidney. (Fig 3 B) 
Endoscopic retrograde cholangiopancreatography (ERCP) was tried to identify the site of leakage and further management, it was not successful, ampullary opening was difficult to access, duodenum and stomach were compressed. Patient was then planned for exploratory laparotomy and cystectomy which was deferred as the cyst wall was not mature $(<3 \mathrm{~mm})$. Patient was then kept on nasojejunal tube to overcome stomach compression, a pig tail catheter was inserted into the pancreatic pseudocyst, around $800 \mathrm{ml}$ of thick purulent discharge was obtained. (Fig $4 \mathrm{~A}$ and B) Patient's general condition gradually improved with adequate bowel rest, higher antibiotics, nutritional supplementation, and aspiration of infected pseudo cyst. The collection obtained from pseudocyst gradually declined and the pig tail catheter was removed on day 12 of placement. Patient was discharged on day 30 of hospital stay with the advice to follow-up regularly.

\section{DISCUSSION}

Pseudocysts of the pancreas are most often associated with alcoholic pancreatitis. Pathogenesis appears to stem from pancreatic duct disturbances due to pancreatitis or trauma accompanied by extravasation of pancreatic secretions. There is demonstrable relation between the cyst and the pancreatic duct in two thirds of patients with pseudocysts. An inflammatory reaction most likely sealed the relation in other third, so that it is not demonstrable. ${ }^{4}$ The suggested management is to observe asymptomatic pseudocysts and those that have been present for less than 6 weeks, as these have a spontaneous resolution rate of 30 to 40 percent. For symptomatic cysts $>5 \mathrm{~cm}$ in diameter or those present for over 6 weeks, internal drainage is recommended because they have a 3 percent chance of resolution and a 57 percent risk of rupture, inflammation, haemorrhage, or obstruction. ${ }^{5}$ Internal drainage to the duodenum, the Roux-en-Y limb of the jejunum, or the posterior wall of the stomach may be done by anastomosis. Given a recurrence rate of 20 percent to 70 percent and the possible risks of infection and fistulisation, percutaneous drainage should be considered when the pseudocyst is infected, located more than $1 \mathrm{~cm}$ from the intestine, or has a cyst wall insufficiently thick to permit anastomosis. ${ }^{6} \mathrm{An}$ individualized, multidisciplinary approach is essential for the management of pseudo pancreatic cysts. Patients with symptomatic, uncomplicated, retro gastric pseudo pancreatic cysts and normal pancreatic ducts are candidates for internal drainage by endoscopy or surgery. Pseudo pancreatic cyst of the head of pancreas, uncinated process, neck, and body may be amenable to trans papillary endoscopic drainage. Pseudo pancreatic cysts that are found in the pancreatic tail should be considered for surgical internal drainage or resection. ${ }^{7}$

Pancreatic duct injury may result from blunt abdominal trauma, ductal lithiasis, ampullary stenosis, or an iatrogenic cause resulting in pancreatic fistula. Pancreatic ascites is caused by a persistent internal fistula within the peritoneum. The presence of necrosis or a pseudocyst increases the risk of pancreatic ascites. Although mild cases of pancreatic ascites spontaneously resolve, there is substantial morbidity and mortality associated with chronic pancreatic ascites and infection. The approach to treatment is to redirect pancreatic fluid with a pancreatic stent away from the leakage to the small intestine, allowing the injury to heal. Medical therapy to minimize pancreatic exocrine secretions and salvage care with surgery is included in adjunct treatment. ${ }^{3}$

In our patient, improvement in acute symptoms was noted with conservative management for acute pancreatitis like bowel rest, higher antibiotics, intravenous fluids, and external percutaneous drainage etc. Pancreatic ascitic fluid gradually subsided with pigtail drainage catheter and other supportive care.

Financial or other competing interests: None.

Disclosure forms provided by the authors are available with the full text of this article at jemds.com.

\section{REFERENCES}

[1] Waller A, Long B, Koyfman A, et al. Acute pancreatitis: updates for emergency clinicians. J Emerg Med 2018;55(6):769-79.

[2] Hot S, Eğin S, Gökçek B, et al. Acute biliary pancreatitis during pregnancy and in the post - delivery period. Ulus Travma Acil Cerrahi Derg 2019;25(3):253-8.

[3] Gapp J, Hoilat GJ, Chandra S. Pancreatic Ascites. In: StatPearls. Treasure Island (FL): StatPearls Publishing 2020 Jan. [Updated 2020 Dec 9].

[4] Habashi S, Draganov PV. Pancreatic pseudocyst. World J Gastroenterol 2009;15(1):38-47.

[5] Eddy JJ, Lynch GE, Treacy DE. Pancreatic pseudocysts in pregnancy: a case report and review of the literature. J Perinatol 2003;23(1):69-72.

[6] Reber HA. The Pancreas. In: Schwartz SI, Shiver GT, Spencer FC, et al. eds. Principles of Surgery. New York, NY: McGraw-Hill 1999: p. 1485-7.

[7] Matsuoka L, Alexopoulos SP. Surgical management of pancreatic pseudocysts. Gastrointest Endosc Clin N Am 2018;28(2):131-41. 